\title{
A Practical and Open Source Implementation of IEC 61850-7-2 for IED Monitoring Applications
}

\author{
Steven M. Blair, Member, IEEE, and Campbell D. Booth
}

\begin{abstract}
A new open source mapping of IEC 61850-7-2 to web services has been defined and implemented. This work is useful for rapidly implementing user interfaces-particularly web-based interfaces-for monitoring and controlling Intelligent Electronic Devices (IEDs) from multiple vendors. The web service mapping has been implemented using the Hypertext Transfer Protocol (HTTP), with a message format in JavaScript Object Notation (JSON). This approach requires a simple and ubiquitous software stack for its implementation, which is a significant advantage over existing client-server communications mappings. The use of an open source paradigm allows for the rapid iteration and refinement of the design, implementation, and testing of the internal details of the proposed protocol stack, in a collaborative manner. These developments are of immediate interest to users of IEC 61850 and are particularly relevant to the IEC 61850 standardization process.
\end{abstract}

Index Terms-Automation and control, code generation, communications, IEC 61850, IED monitoring, power system protection, rapid-prototyping, user interfaces.

\section{INTRODUCTION}

$\mathbf{T}$ HE secure and standardized access to power system data will enable smarter grids [1]. To facilitate system integration and to ensure a consistent user experience for engineers who use substation automation tools, a simple method is needed for accessing the data stored in Intelligent Electronic Devices (IEDs) [2]. One of the benefits of the design of the IEC 61850 series of standards is the ability to define new mappings of the IEC 61850-7-2 services to a specific protocol or technology. This paper describes a new Specific Communication Service Mapping (SCSM) which provides client-server communications, such as accessing an IED data attribute value, between two or more IEDs.

The main purpose of the proposed mapping is to facilitate the exchange of data between multiple IEDs, and thereby to significantly simplify the process of creating IED monitoring applications. This has been achieved by the design of a mapping of IEC 61850-7-2 to the Hypertext Transfer Protocol (HTTP) using JavaScript Object Notation (JSON).

\section{MethoD}

Fig. 1 illustrates the proposed protocol stack, and compares this with the existing Manufacturing Message Specification (MMS) mapping defined in IEC 61850-8-1 [3]. The proposed stack is significantly simpler than MMS, and uses technologies which are ubiquitous: the stack can readily be implemented

The authors are with the Institute for Energy and Environment, Department of Electronic and Electrical Engineering, University of Strathclyde, Glasgow, G1 1XW, UK (e-mail: steven.m.blair@strath.ac.uk).

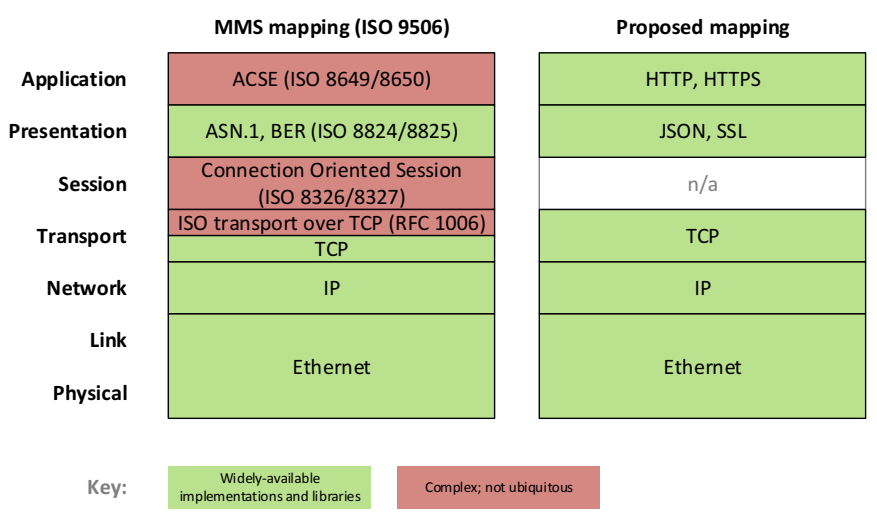

Fig. 1. Comparison of protocol stacks

on most hardware platforms and suitable software libraries are available in most programming languages. This method is highly practical because it uses ubiquitous protocols and technologies. No specialist software is required for interacting with IEDs, unlike protocols such as MMS and Object Linking and Embedding for Process Control (OPC) [4]. Although commercial and open source MMS implementations are available, it is challenging and complex to implement a complete MMS stack on a wide variety of platforms, including personal computers (for multiple operating systems), microcontrollers, smartphones, and tablets.

The interface for accessing IED data adopts the representational state transfer (REST) methodology presented in [5]. HTTP requests typically use the following format:

GET https:// address: port $/<$ command $>/<$ LD $>/<$ ObjectRef $>$

where " $<\mathrm{LD}>\mid<$ ObjectRef $>$ "specifies the ObjectReference as defined in IEC 61850-7-2. Fig. 2 further illustrates examples of using the proposed mapping. For example, to implement the GetDataValues service defined in [6], a client sends the HTTP "GET" method to a server to retrieve a specific data attribute value. As shown in Fig. 2, the client supplies the "C1/LN0.NamPlt.configRev" ObjectReference within the URI and the server responds with the value of "Rev 3.45" which is encoded within a JSON object (assuming a valid ObjectReference was supplied).

The use of JSON as the message format is particularly suited to the creation of user interfaces which are web applications written in the JavaScript programming language, compared with the XML-based approach used in IEC 61400-25-4 [7]. The message format can be formally enforced by a JSON schema to facilitate automated code generation and message validation [8]. 


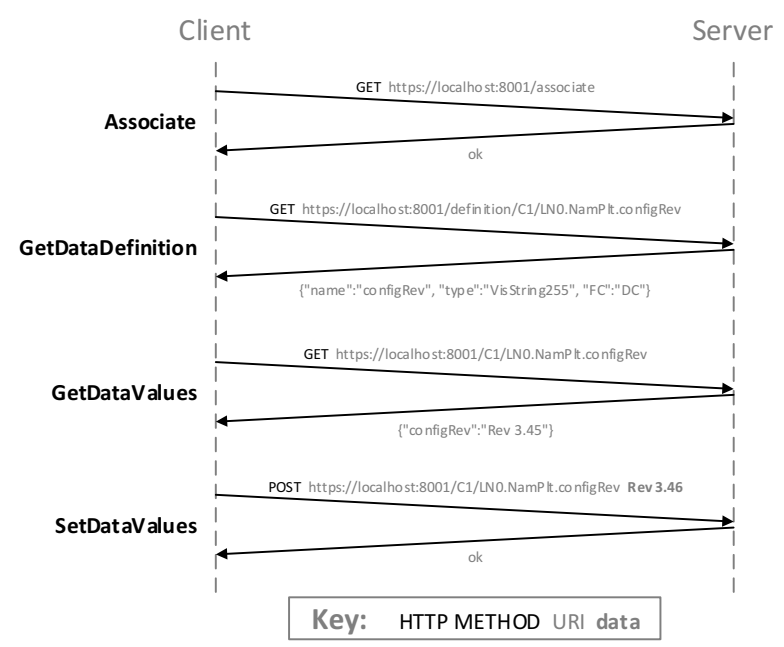

Fig. 2. Example of HTTP and JSON mapping for data access

\section{PROOF-OF-CONCEPT IMPLEMENTATION}

An example implementation has been created using the open source "rapid61850" tool [8] and is available at [9] with documentation. Using a standardized configuration file, the data model is generated automatically along with all metadata required for the data model to be "self-describing" at runtime. This provides a mechanism for rapid-prototyping an IED which supports both client-server communications (using the proposed mapping) and publisher-subscriber communications (using GOOSE and Sampled Values). The generated C code is also IED-specific, offering benefits in run-time performance, and is designed to be independent of the hardware platform. It is also possible for a personal computer to instantiate multiple IEDs simultaneously using multiple execution threads, to facilitate tasks such as IED black-box testing or testing the coordination of multiple IEDs.

At present, the stack implements the following services: Associate, Release, Abort, GetDataDirectory, GetDataDefinition, GetDataValues, and SetDataValues. A protocol converter, or gateway, has been created to convert the proposed HTTP-based interface to MMS. This allows interaction with existing commercial IEDs using the proposed mapping. HTTP authentication and encryption using TLS/SSL ensure secure access to IED data. The implementation of the GetDataDirectory and GetDataDefinition services permits the discovery of IED capabilities at run-time, and thereby allows for the provision of a suitable user interface which is dependent on IED capability.

\section{Application: Multi-Vendor IED Monitoring}

Using the open source software described in Section III, a multi-vendor IED monitoring application has been developed. The user interface has been rapid-prototyped using emerging standards for Web Components [10] and is shown in Fig. 3. Critical IED information, for multiple vendors, are displayed conveniently and consistently.

\section{CONCLUSIONS}

This paper has proposed a new approach for IED clientserver communications which is compliant with IEC 61850-

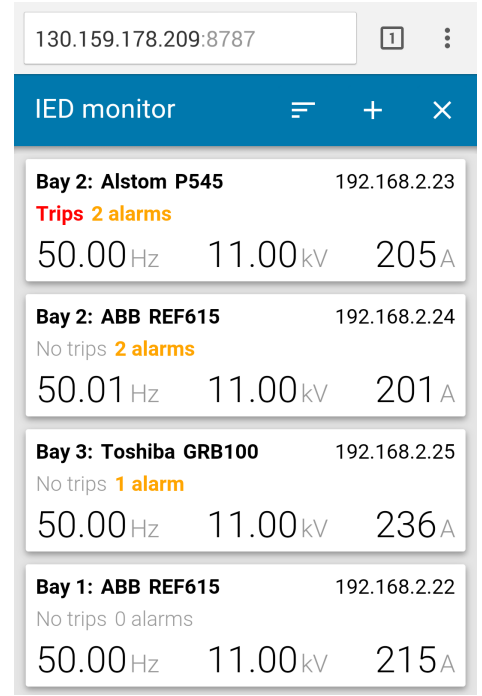

Fig. 3. User interface for multi-vendor IED monitoring, within a smartphone web browser

7-2. Compared to existing methods, the proposed approach is simple to implement, uses ubiquitous technologies, and is particularly suited to the development of web-based IED monitoring applications. Furthermore, the proposed mapping does not compromise security. The open source software is readily available for use in research and development activities, and these contributions can be used and improved by others.

\section{REFERENCES}

[1] C. Gellings, "The Future of the Power Grid," 2014 [Online]. Available: http://spectrum.ieee.org/podcast/energy/the-smartergrid/clark-gellings-the-future-of-the-power-grid

[2] L. Li, X. Zhao, and G. Huon, "Special Report for CIGRE Study Commitee B5 (Protection and Automation)," CIGRE, Tech. Rep., 2014

[3] IEC TC 57, "Communication networks and systems for power utility automation Part 8-1: Specific communication service mapping (SCSM)Mappings to MMS (ISO 9506-1 and ISO 9506-2) and to ISO/IEC 88023 ," 2011.

[4] F. Coffele, S. M. Blair, C. D. Booth, J. Kirkwood, and B. Fordyce, "Demonstration of Adaptive Overcurrent Protection Using IEC 61850 Communications," in CIRED, 2013.

[5] A. B. Pedersen, E. B. Hauksson, P. B. Andersen, B. Poulsen, C. Traeholt, and D. Gantenbein, "Facilitating a Generic Communication Interface to Distributed Energy Resources: Mapping IEC 61850 to RESTful Services," in 2010 First IEEE International Conference on Smart Grid Communications. IEEE, Oct. 2010, pp. 61-66.

[6] IEC TC 57, "Communication networks and systems for power utility automation Part 7-2: Basic information and communication structure Abstract communication service interface (ACSI), 2010.

[7] IEC TC 88, "IEC 61400-25-4 - Mapping to communication profile," 2008.

[8] S. M. Blair, F. Coffele, C. D. Booth, and G. M. Burt, "An Open Platform for Rapid-Prototyping Protection and Control Schemes with IEC 61850," IEEE Transactions on Power Delivery, vol. 28, no. 2, pp. 1103-1110, 2013.

[9] S. M. Blair, "rapid61850," 2012. [Online]. Available: https://github.com/stevenblair/rapid61850

[10] The World Wide Web Consortium (W3C), "Introduction to Web Components," 2013. [Online]. Available: http://www.w3.org/TR/componentsintro/ 